\title{
Low-power variable-length fast Fourier transform processor
}

\author{
Y.-T. Lin, P.-Y. Tsai and T.-D. Chiueh
}

\begin{abstract}
Fast Fourier transform (FFT) processing is one of the key procedures in the popular orthogonal frequency division multiplexing (OFDM) communication systems. Structured pipeline architectures and low power consumption are the main concerns for its VLSI implementation. In the paper, the authors report a variable-length FFT processor design that is based on a radix-2/4/8 algorithm and a single-path delay feedback architecture. The processor can be used in various OFDM-based communication systems, such as digital audio broadcasting (DAB), digital video broadcasting-terrestrial (DVB-T), asymmetric digital subscriber loop (ADSL) and very-high-speed digital subscriber loop (VDSL). To reduce power consumption and chip area, special current-mode SRAMs are adopted to replace shift registers in the delay lines. In addition, techniques including complex multipliers containing three real multiplications, and reduced sine/cosine tables are adopted. The chip is fabricated using a $0.35 \mu \mathrm{m}$ CMOS process and it measures $3900 \mu \mathrm{m} \times 5500 \mu \mathrm{m}$. According to the measured results, the 2048-point FFT operation can function correctly up to $45 \mathrm{MHz}$ with a $3.3 \mathrm{~V}$ supply voltage and power consumption of $640 \mathrm{~mW}$. In low-power operation, when the supply voltage is scaled down to $2.3 \mathrm{~V}$, the processor consumes $176 \mathrm{~mW}$ when it runs at $17.8 \mathrm{MHz}$.
\end{abstract}

\section{Introduction}

The fast Fourier transform plays an important role in many digital signal processing (DSP) systems. Recent advances in semiconductor processing technology have enabled the deployment of dedicated FFT processors in applications such as telecommunications, speech and image processing. Specifically, in the OFDM communication systems, FFT and inverse FFT (IFFT) play a very important role. The OFDM technique, due to its effectiveness in overcoming adverse channel effects $[1,2]$ as well as spectrum utilisation, has become widely adopted in wireline and wireless communication standards.

The OFDM technique has been adopted in several standards like digital audio broadcasting (DAB) [3], digital video broadcasting-terrestrial (DVB-T) [4], asymmetrical digital subscriber line (ADSL) [5] and very-high-speed digital subscriber line (VDSL) [6]. Therefore, efficient and low-power VLSI implementation of FFT processors is essential for successful deployment of these OFDM-based systems. According to the standards of DAB, DVB-T, ADSL and VDSL, various FFT sizes are required, as shown

(C) IEE, 2005

IEE Proceedings online no. 20041224

doi: 10.1049/ip-cdt:20041224

Paper first received 16th March and in revised form 19th October 2004

Y.-T. Lin was with the Department of Electrical Engineering, National Taiwan University and is now with the Mediatek, Hsin-Chu, Taiwan, Republic of China

P.-Y. Tsai is with the Department of Electrical Engineering, National Taiwan University, Taipei, Taiwan 10617, Republic of China

T.-D. Chiueh is with the Graduate Institute of Electronics Engineering and the Department of Electrical Engineering, National Taiwan University

E-mail: chiueh@cc.ee.ntu.edu.tw in Table 1. From this Table, it is clear that a variable-length FFT hardware is a crucial module in the low-cost solution of the above communication systems.

The Cooley-Tukey $N$-point FFT algorithm requires $\mathrm{O}(N \log N)$ computations, which is a huge saving over direct computation of the discrete Fourier transform (DFT). However, hardware implementation of the algorithm is both computational intensive, in terms of arithmetic operations, and communication intensive, in terms of data swapping. For real-time processing of FFT, $\mathrm{O}(\log N)$ arithmetic operations are required per sample cycle. High speed real-time processing can be accomplished in two different ways. In the conventional general-purpose digital signal processor (DSP) approach, the computation is carried out by a single processor driven to a high clock frequency, which is $\mathrm{O}(\log N)$ times the data sample frequency. In the application-specific parallel or pipelined processor approach, the required operations are performed at the clock frequency equivalent to the sample frequency, and this approach usually consumes less power.

In this paper, we aim to implement a low-power variablelength FFT processor. To this end, we adopt several optimisation techniques in the circuit design to accomplish an area- and power-efficient pipelined FFT processor.

\section{Pipelined FFT processor architecture}

\subsection{Radix-2 FFT architecture}

The radix-2 multi-path delay commutator [7] is a pipelined implementation of the radix-2 FFT algorithm. A radix-2 multi-path delay commutator architecture with $N=8$ is shown in Fig. 1. The input sequence is divided into two parallel data streams by a commutator and then, with proper scheduling for two streams, butterfly operation in a processing element (PE) and twiddle factor multiplication are executed. In total, $\left(\log _{2} N-2\right)$ multipliers, $\log _{2} N$ radix- 2 butterfly units, and $(3 N / 2)-2$ delay elements are 
Table 1: FFT sizes in several OFDM communication systems

\begin{tabular}{ll}
\hline Communication system & FFT size \\
\hline ADSL & 512 \\
VDSL & $8192,4096,2048,1024,512$ \\
DAB & $2048,1024,512,256$ \\
DVB-T & 8192,2048
\end{tabular}

required. With a proper input buffering scheme, the processing element can work at $100 \%$ utilisation.

Radix-2 single-path delay feedback architecture (shown in Fig. 2) utilises the delay elements more efficiently by sharing the same storage between the butterfly outputs and inputs [8]. A single data stream goes through the multiplier at every stage. This architecture has the same number of processing elements (PEs) and multipliers as needed in the radix-2 multi-path delay commutator architecture, albeit only $N-1$ delay elements. Note that the butterfly units and multipliers work at $50 \%$ utilisation since half of the time they are bypassed.

\subsection{Radix-2/4/8 FFT algorithm and architecture}

The $N$-point DFT is formulated as

$$
X_{k}=\sum_{n=0}^{N-1} x_{n} W^{n k}, \quad k=0,1,2, \ldots, N-1
$$

where $W^{n k}=e^{-j 2 \pi \frac{n k}{N}}$. The basic concept underlying the radix-2 FFT algorithm is the use of symmetry between the twiddle factors $W^{n k}$ and $W^{n k+N / 2}\left(W^{n k}=-W^{n k+N / 2}\right)$. Another symmetry of twiddle factors $\left(W^{n k+N / 4}=-\right.$ $\left.W^{n k+3 N / 4}=-j W^{n k}\right)$ has been utilised to reduce the number of complex multiplications by $\pm j$ in radix -4 and split radix algorithms.

Exploiting twiddle factor symmetry further, the multiplication by the twiddle factors of $W^{N / 8}, W^{3 N / 8}, W^{5 N / 8}$ and $W^{7 N / 8}$ can be further simplified since their real and imaginary parts have equal magnitude. The complex multiplications by these four twiddle factors can be formulated as:

$$
\begin{aligned}
(a+j b) W^{N / 8} & =-(a+j b) W^{5 N / 8} \\
& =\frac{\sqrt{2}}{2}[(a+b)+j(b-a)] \\
(a+j b) W^{3 N / 8} & =-(a+j b) W^{7 N / 8} \\
& =\frac{\sqrt{2}}{2}[(b-a)-j(a+b)]
\end{aligned}
$$

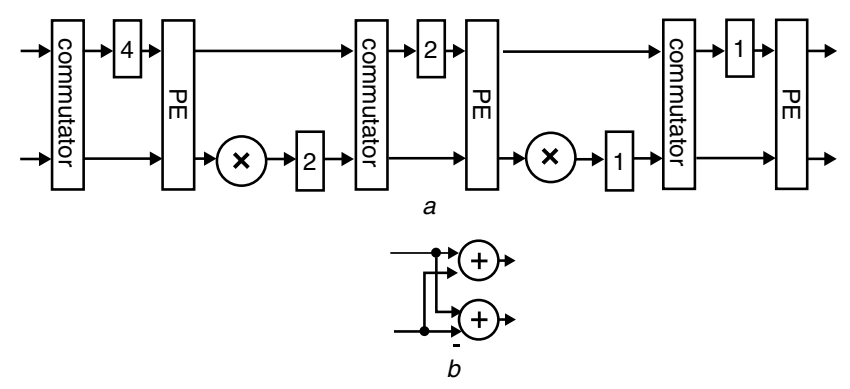

Fig. 1 Radix-2 multi-path delay commutator FFT architecture, and $P E$
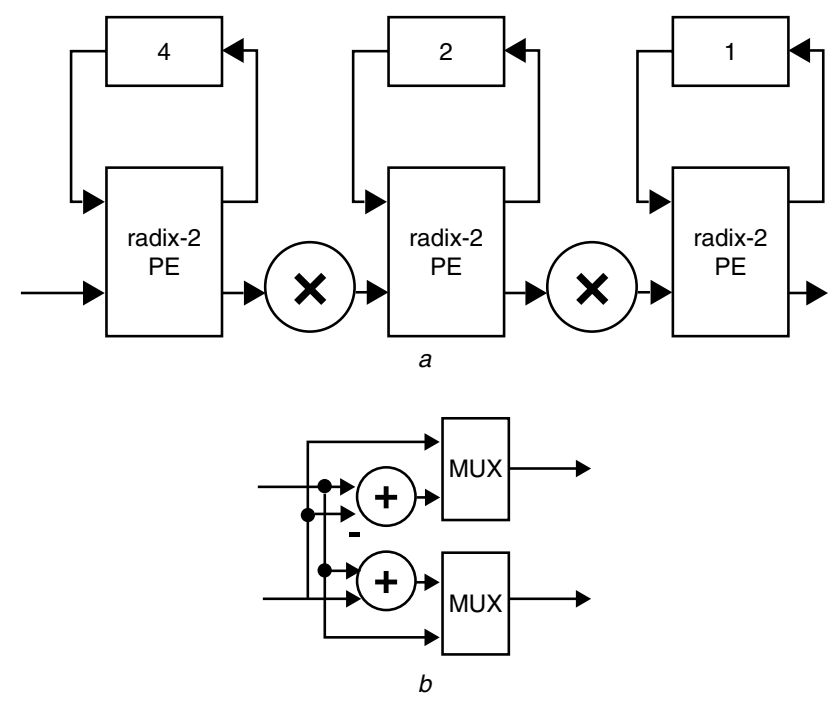

Fig. 2 Radix-2 single-path delay feedback FFT architecture, and $P E$

Note that these complex multiplications can be realised by two real multiplications and two additions.

The signal flow graph (SFG) of the radix-2/4/8 FFT algorithm is shown in Fig. 3 [9]. Instead of one single butterfly, the radix $-2 / 4 / 8$ algorithm implements the radix -8 butterfly using three radix- 2 stages. Therefore its SFG is equivalent to that of the radix $-2^{3}$ algorithm [10]. Note that by modifying the radix-2 single-path delay feedback FFT architecture, a radix$2 / 4 / 8$ architecture was proposed in [9]. There are three types of basic processing elements, called PE1, PE2 and PE3, and each processes one FFT stage. The architecture is made up of a repeated cascade of PE1, PE2, PE3 and a general complex multiplier for twiddle-factor multiplication. The number of delay elements needed decreases by half in every stage. The block diagrams of these three types of processing elements are illustrated in Fig. 4.

\subsection{Proposed variable-length FFT processor architecture}

At the architecture level, to reduce power consumption and chip area, it is desirable to adopt the FFT algorithm which has least computational complexity and the architecture that corresponds to less hardware complexity. The block diagram of the proposed variable-length FFT processor based on the radix-2/4/8 single-path delay feedback architecture is depicted in Fig. 5. The proposed processor can perform FFT operations of three different lengths: 2048point, 1024-point and 512-point. To accommodate different numbers of FFT stages, the first two stages are radix-2 PEs, which have the same structure as the PE3 unit in the radix$2 / 4 / 8$ architecture, and each of the following three blocks is made up of a set of PE1, PE2 and PE3 and a twiddle-factor multiplier. If 512-point FFT is executed, then input signals skip the first two stages through the control of the multiplexer, MUX2. If a 1024-point FFT is performed, the first stage is bypassed through MUX1.

\section{Architecture considerations}

\subsection{Comparison of FFT architectures}

Over the years, various FFT architectures have been proposed with a view to providing speedy and efficient implementation of the all-important FFT operation. In Table 2, we list some computational features the radix$2 / 4 / 8$ FFT architecture used in the proposed IC and 


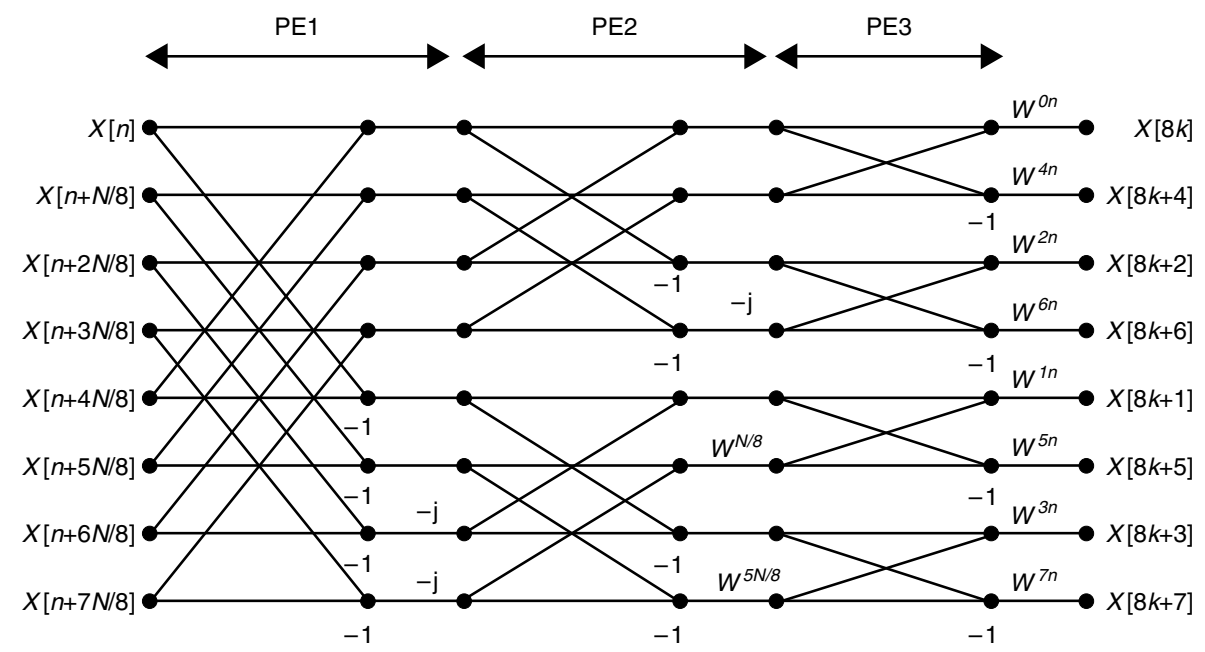

Fig. 3 Signal flow graph of the radix-2/4/8 FFT algorithm
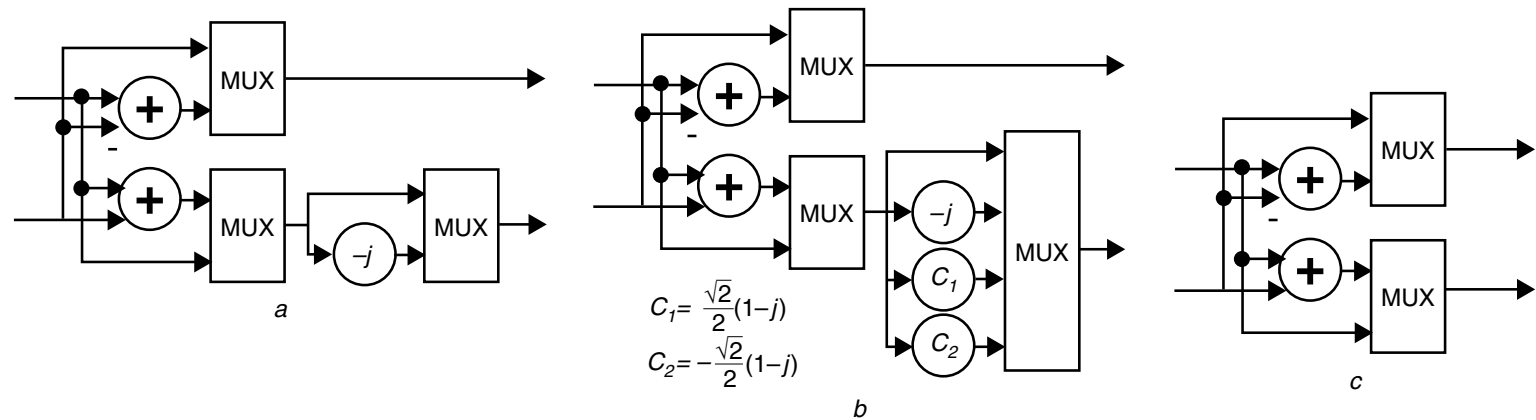

Fig. 4 Block diagrams of the PE units in the radix-2/4/8 architecture

$a$ PE1

$b$ PE2

$c$ PE3
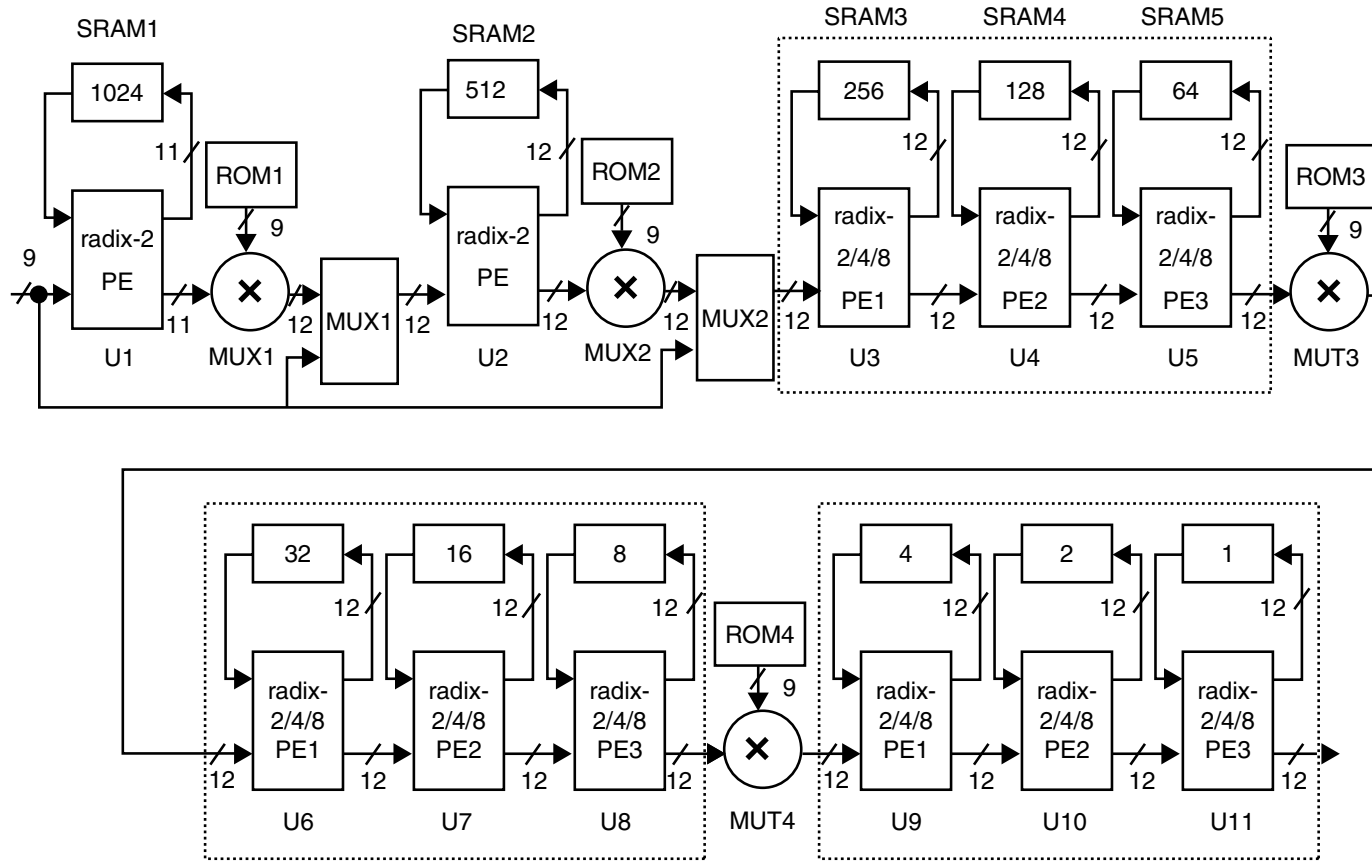

Fig. 5 Block diagram of proposed variable-length FFT processor

several other recent architectures. In the Table, we compare their computational complexity and memory requirements. It is apparent that the number of nontrivial complex multiplications decreases as the radix gets higher.
In addition, in bit-parallel operation, higher-radix algorithms also have better hardware utilisation in multipliers. As to the adders in butterfly units, if the higher radix butterfly operation is implemented by concatenating radix- 2 


\begin{tabular}{|c|c|c|c|c|}
\hline & \multicolumn{2}{|l|}{ Bit-parallel } & \multicolumn{2}{|l|}{ Digit-serial } \\
\hline & Proposed chip & He \& Torkelson [10] & Hui et al. [11] & Chang \& Parhi [12] \\
\hline Radix & radix- $2 / 4 / 8$ & radix-4 & radix-4 & radix- 4 \\
\hline Data flow & feedback & feedback & feedforward & feedforward \\
\hline \multirow[t]{2}{*}{ Complex adder utilisation } & $2 \log _{2} N$ & $4 \log _{4} N$ & $12 \log _{4} N$ & $8\left(\log _{4} N+1\right)$ \\
\hline & $50 \%$ & $50 \%$ & $100 \%$ & $\sim 100 \%$ \\
\hline \multirow[t]{2}{*}{ Complex multiplier utilisation } & $\log _{8} N-1$ & $\log _{4} N-1$ & $3\left(\log _{4} N-1\right)$ & $3\left(\log _{4} N-1\right)$ \\
\hline & $87.5 \%$ & $75 \%$ & $100 \%$ & $100 \%$ \\
\hline Data memory & $N-1$ & $N-1$ & $2.5 N$ & $1.18 N$ \\
\hline Twiddle factor ROM & $0.25 N$ & $N$ & $N$ & $0.5 N$ \\
\hline
\end{tabular}

butterfly units, such as in [10], then only $50 \%$ adder utilisation can be achieved. Note that in the digit-serial architectures [11, 12], the word-length of the data in adders and multipliers is reduced to one digit and thus fewer full adders are required. On the other hand, to achieve almost $100 \%$ utilisation in adders and multipliers, the word-length of the signals in these two architectures must be restricted to match the throughputs of the radix -4 commutator -4 digits in these cases. Nevertheless, the occupied area of one complex multiplier overwhelms the area of one complex adder. Thus, a great saving in the cost of silicon can be accomplished with fewer complex multipliers.

A feedback FFT architecture needs the least amount of data memory, in the size of $N-1$. On the other hand, a feed-forward architecture requires more memory elements, as in $[11,12]$. Other memory blocks are the look-up-table ROMs that store twiddle factors. If the number of nontrivial complex multiplications is decreased, then there are fewer twiddle-factor ROMs. The twiddle-factor ROM for the first multiplier stores twiddle factors with a phase spacing of $2 \pi / N$. In the later stages, the phase spacing increases. If the symmetry of the sine/cosine function is further exploited, more saving in ROM size can be had. In the proposed chip, the twiddle-factor ROMs store only one-eighth cycle of the sine/cosine waveforms and we take advantage of the symmetry of all the twiddle factors instead of the redundancy within each group of $W^{n}, W^{2 n}$ and $W^{3 n}$, for $n=0,1, \ldots, N / 4$, in the radix-4 algorithm as in [12], and consequently a smaller ROM table is built.

In summary, the radix- $2 / 4 / 8$ algorithm can bring forth a variable-length FFT processor with the least overall hardware complexity. Although its adder and multiplier utilisation is not as good as other architectures, we decide to adopt this architecture because it strikes a balance between hardware complexity and computational efficiency.

\subsection{Complex multiplier against CORDIC}

The CORDIC algorithm has been used for the twiddle factor multiplication in FFT processors due to its efficiency in vector rotation [13]. In this sub-Section, we evaluate and compare the performance and complexity of a CORDIC and a complex multiplier in phase rotation. In Table 3 , the conventional CORDIC algorithm refers to the radix-2 CORDIC, and radix-2/4 CORDIC refers to the work in [14] that enhances operation speed and reduces $25 \%$ of the micro-rotation stages. The complex multiplier used in the proposed chip consists of three multiplications and five additions [15]. To make a fair comparison, we set the precision to 16 bits in all algorithms. To avoid rounding error propagation [14, 16], 19 bits are allocated in the data path of the CORDIC-based architectures.
In the conventional CORDIC algorithm, a ROM table that stores the rotation sequences with $N / 4$ 16-bit words in the range of $[0, \pi / 2]$ is used. Two 19-bit adders are required in each micro-rotation stage and the conventional CORDIC architecture needs $2 \times 16 \times 19=608$ full adders for 16 micro-rotation stages. Additional constant multiplication by 0.100110110111010 as the scaling factor is performed in the final scaling stage and it needs $2 \times 9 \times 19$ adders. Without pipelining, its critical path delay is $19 \times 16$ times the full adder delay $\left(\mathrm{T}_{\mathrm{FA}}\right)$ in the 16 micro-rotation stages plus 28 $\mathrm{T}_{\mathrm{FA}}$ in the scaling stage.

In [14], the ROM table is further reduced to $N / 8$ words with 23 bits per word due to the higher radix adopted in the later stages. According to the authors, each stage is based on a similar cell with a 4-2 adder/subtracter using two-level carry-save adders (CSA) and redundant arithmetic representation intended to improve the performance. Two registers are used to buffer the intermediate sum and carry in each stage. Meanwhile two full adders are connected to perform the 4-2 compression. As a result, a total of $2 \times 19 \times$ $17 \times 2$ full adders are provided in the 17 stages including additional micro-rotation-repetition stages and 2 scaling stages. Because of pipelining in every stage, the critical path delay is reduced to about $2 \mathrm{~T}_{\mathrm{FA}}$ with a penalty of a large number of $(17 \times 19 \times 2 \times 2)$ pipeline registers. Actually, its CORDIC outputs are still in the form of redundant arithmetic representation and will be transformed back to the binary format after butterfly operation by carry-lookahead adders.

In the proposed chip, complex multiplication consists of five real additions and three real multiplications. The real addition is implemented by carry-selected adders with a maximum delay of about $8 \mathrm{~T}_{\mathrm{FA}}$ and each utilises 30 full adders in the first 16-bit addition and 63 full adders in the last 33-bit addition. Because Wallace tree multipliers are adopted for the three $16 \times 17$ multiplications, the critical path delay is reduced to $7 \mathrm{~T}_{\mathrm{FA}}$. One $16 \times 17$ Wallace tree multiplier needs about 280 full adders, and two pipeline stages are inserted before and after the multiplication.

From Table 3, we can see that the CORDIC algorithm may be too slow without pipelining. On the other hand, Wallace tree multiplication reduces the critical path delay of the complex multiplier approach. Considering all aspects of speed-area tradeoff and that the application of the FFT processor is low power consumption rather than high speed, we use the complex multiplier for twiddle factor multiplication.

\section{Circuit design}

To serve as a key component in OFDM communication systems, the variable-length FFT processor must be designed to reduce its power consumption as well as chip 
Table 3: Comparison between CORDIC algorithms and simplified complex multiplication

\begin{tabular}{llll}
\hline & Conventional CORDIC & Radix-2/4 CORDIC [14] & Complex multiplier \\
\hline Word length of data paths & 19 & 19 & 16 \\
Stages & 17 & 17 & - \\
ROM table size & $16 \times / \mathrm{N} / 4$ & $23 \times / \mathrm{N} / 8$ & $16 \times \mathrm{N} / 4$ \\
Adders & $2 \times 16 \times 19+2 \times 9 \times 19=950$ & $2 \times 17 \times 19 \times 2=1292$ & $3 \times 30+3 \times 280+2 \times 63=1056$ \\
Critical path delay & $\sim(16 \times 19+28) \mathrm{T}_{\mathrm{FA}}$ & $\sim 2 \mathrm{~T}_{\mathrm{FA}}$ & $\sim 8 \mathrm{~T}_{\mathrm{FA}}$ \\
Number of pipeline stages & 1 & 18 & 3 \\
Pipeline registers & 0 & $17 \times 19 \times 2 \times 2=1292$ & $17 \times 6+33 \times 3=201$ \\
\hline
\end{tabular}

area. The power consumption is usually determined by the dynamic power, which is given by

$$
P_{d}=\alpha C_{L} V_{d d}^{2} f_{c l k}
$$

where $\alpha$ is the switching probability, $C_{L}$ is the load capacitance, $V_{d d}$ is the supply voltage and $f_{c l k}$ is the clock rate. To achieve low power consumption, several techniques, such as word-length minimisation, special-RAMbased delay line, complex multiplication re-formulation and small look-up-table ROMs are employed. These techniques and the associated circuits will be described in the following.

\subsection{Word-length minimisation}

In the design of this application-specific variable-length FFT processor, the word lengths of various signals are minimised according to their respective signal-to-noise ratio (SNR) requirements. To decide the optimal word length, input waveforms with Gaussian noise are fed to the FFT with fixed-point arithmetic implementation. The frequencydomain FFT output signals are obtained and the output signal-to-noise ratio (SNR) is computed. Figure $6 a$ shows the output SNR against the FFT input word length under different input SNR conditions. Accordingly, the word length of the input is set to 9 bits. As to the precision of the sine and cosine tables, the output SNR against the word length of the twiddle factors is shown in Fig. $6 b$ when the input signal has an SNR of $30 \mathrm{~dB}$. A word length of 9 bits is thus chosen for the twiddle factors. The word-length minimisation process then goes on module by module and the word lengths of all signals in the processor are determined, and are labelled in Fig. 5.

\subsection{RAM-based delay line}

A single-path delay feedback FFT processor needs several long and wide delay lines. Conventionally, delay lines are mostly implemented in shift registers, made up of cascades of data registers, as shown in Fig. 7a. At each clock edge, all data move forward in a lock-step fashion and approximately half of the registers change states, wasting much power. To save power and chip area, SRAM has been utilised to replace the shift registers. Since the read and write operations must be performed in one clock cycle, intuitively a dual-port memory is required. Two single-port SRAMs are adopted in [17], and the authors claimed that a single-port memory can save $33 \%$ in area over a dual-port memory. Here we use one single-port SRAM as shown in Fig. 7b. The SRAM is designed manually. In the first half clock cycle, the read operation is performed while the write operation follows in the next half clock cycle. To prevent the data access of the SRAM becoming critical paths, two registers, one before the PE and the other after, are inserted. Furthermore, a ring counter is used instead of the conventional address decoder since data to and from the SRAM are accessed sequentially. To further conserve power consumption, true-single-phase-clock (TSPC) flipflops are used in the ring counters.

\subsection{Current-mode SRAM}

The current-mode technique has been used in reading SRAM cell contents. It has been proposed that the currentmode technique can also be applied to the writing operation of SRAM so as to further reduce power consumption [18]. This is because voltage swings of the SRAM bit lines and data lines can be kept very small in the current-mode $\mathrm{read} /$ write operations and thus the dynamic power dissipation can be significantly decreased.
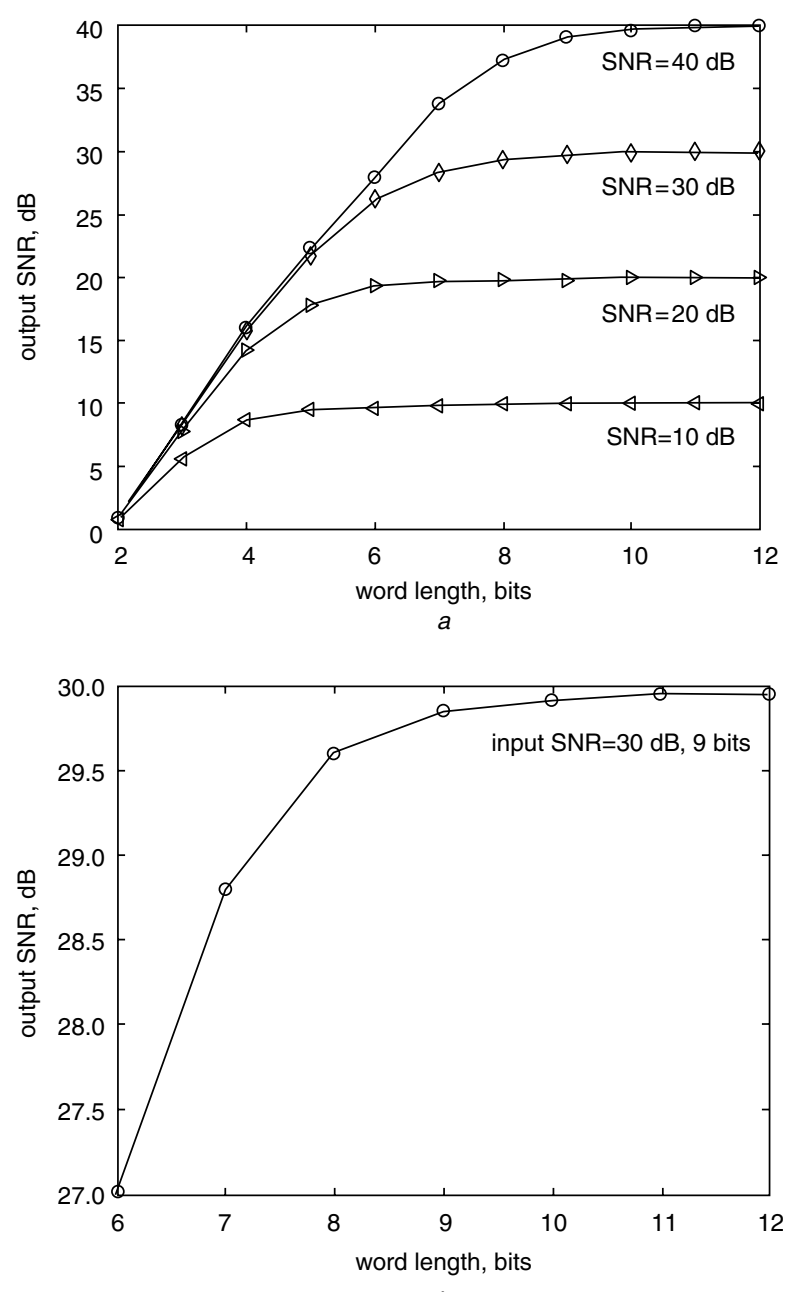

b

Fig. 6 Output SNR against word length of the FFT processor input and of twiddle factor 

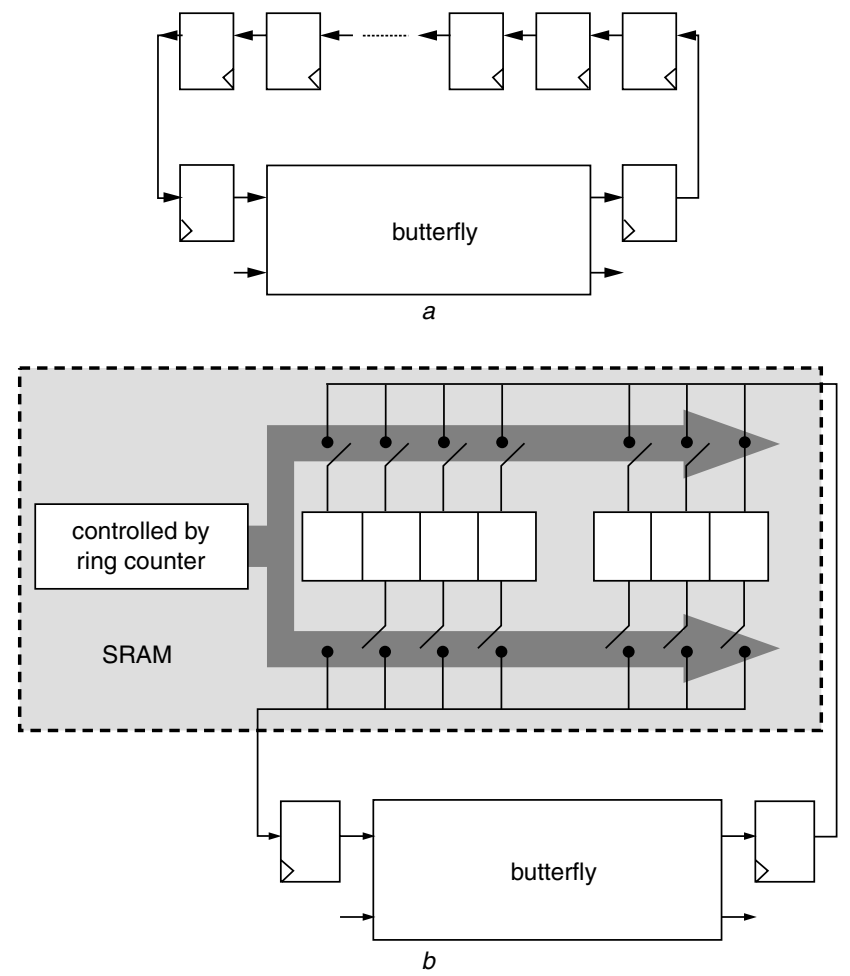

Fig. 7 Conventional shift-register-based delay line and proposed SRAM-based delay line

The current-mode SRAM cell used is based on that proposed in [18], and it consists of seven transistors, one more than the conventional 6-transistor SRAM cell, and it is depicted in Fig. $8 a$. An extra transistor, $M_{e q}$, is inserted to equalise the output voltages of the two inverters before each write operation, and therefore a small current difference can be sensed through access transistors controlled by the
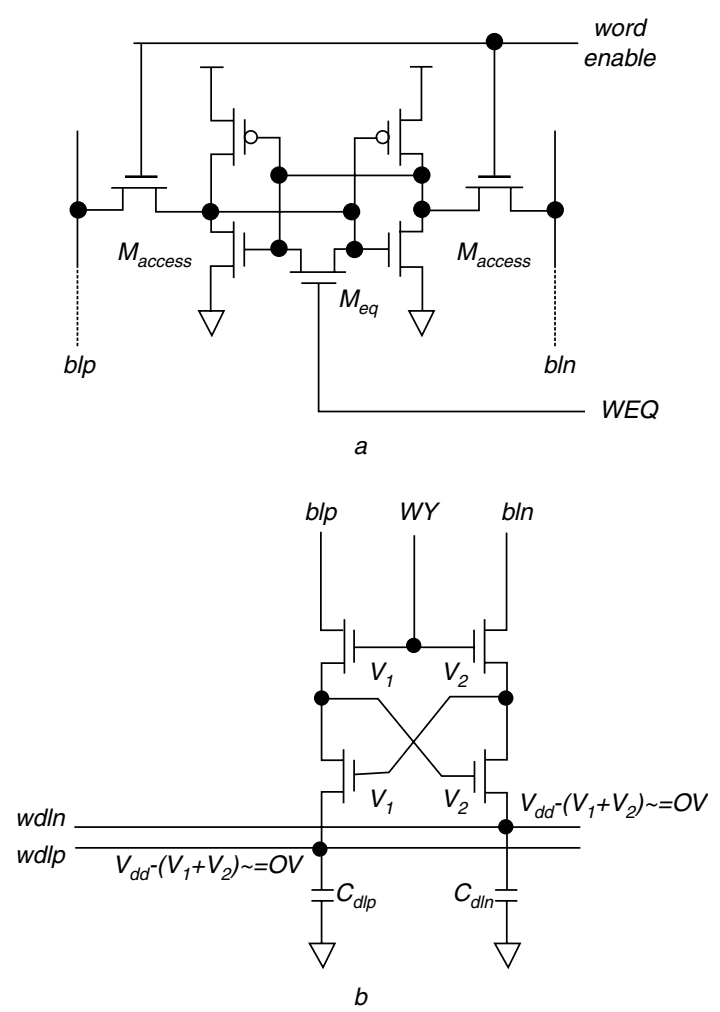

Fig. 8 Schematic diagrams of proposed 7T current-mode SRAM memory cell and of SRAM write circuitry using N-type current conveyor word-line enable signal and amplified by the inverters. When $M_{e q}$ is off, the cell performs as the conventional 6T SRAM memory cell.

During write access, a current difference, $\Delta I$, appears on the write data lines $\boldsymbol{w d l p}$ and $\boldsymbol{w d l n}$. The $N$-type current conveyor (shown in Fig. $8 b$ ) is enabled by the signal $\mathbf{W Y}$. Then the currents are conveyed to the bit lines $\boldsymbol{b l p}$ and $\boldsymbol{b l n}$ without attenuation. Because the control signal $\boldsymbol{W Y}$ is enabled, a virtual short circuit exists between the write data lines $\boldsymbol{w d l p}$ and $\boldsymbol{w d l n}$. Both the voltages at $\boldsymbol{w d l p}$ and $\boldsymbol{w d l n}$ are equal to $V_{D D}-\left(V_{1}+V_{2}\right)$, which can be designed to approach the ground voltage. Thus the voltage swing on data lines can be kept as small as possible. The read operation in this SRAM is implemented by a sense amplifier, which has the same structure as the conventional SRAM, and a column decoder. As in conventional SRAM, a read access starts with the word line being enabled and the pair of bit lines driven by a differential current, which is then steered to the sense amplifier, where the data are sensed and buffered.

\subsection{Complex multiplication and twiddle- factor $R O M$}

In the proposed FFT processor, due to the radix-2/4/8 algorithm, each complex multiplication of $W^{N / 8}, W^{3 N / 8}$, $W^{5 N / 8}$ and $W^{7 N / 8}$ is reduced to two real multiplications by the constant $\sqrt{2} / 2$ as shown in (2) and (3), which can be further simplified to shift and add operations [9], since

$$
\frac{\sqrt{2}}{2}=0.70710678=2^{-1}+2^{-3}+2^{-4}+2^{-6}+2^{-8}
$$

In addition, four twiddle-factor complex multipliers as well as four twiddle-factor ROMs are needed in the FFT processor. A simplified complex multiplication formula is given by [15]

$$
\begin{aligned}
(A+j B) *(C+j D)= & {[C *(A-B)+B *(C-D)] } \\
& +j[D *(A+B)+B *(C-D)]
\end{aligned}
$$

Note that only three real multiplications and five real additions are needed. With one less real multiplication operation than the original complex multiplication formula, the complex multiplier saves die area as well as power consumption. The partial products of these three multiplications are first generated and then fed into a Wallace tree. The five additions are implemented using carry-select adders for high-speed consideration.

The twiddle-factor ROMs provide sine and cosine values for various phases. The even and odd symmetry in the phase and amplitude of these two functions can be exploited to save the size of a twiddle-factor ROM. Following the approach in [19], only sine and cosine values of phases from 0 to $\pi / 4$ are stored. The sample index for the FFT operation, counting from 0 to $N-1$, is used to generate this phase that addresses all the twiddle-factor ROMs. The first three MSBs of the phase are used as the control signals to synthesise the correct sine/cosine value from the outputs of the two oneeighth-cycle tables addressed by the remaining bits of the phase. The detailed circuit diagram of this twiddle-factor ROM is illustrated in Fig. 9.

\section{Experimental result}

The whole chip, except for the SRAM modules, was designed by a gate-level hardware description language. The critical path lies in the complex multiplier. The layout of the SRAM modules containing the ring counters, timing control units as well as the SRAM cells are all designed 


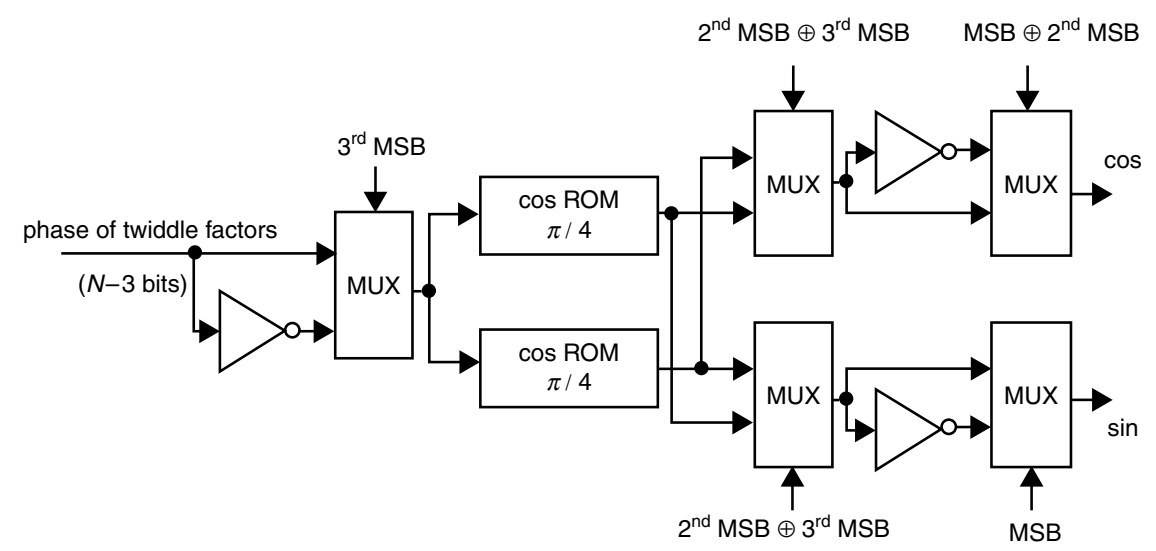

Fig. 9 Block diagram of twiddle-factor ROM

manually. This proposed FFT processor is fabricated using a $0.35 \mu \mathrm{m}$ CMOS process. The chip's die photo is shown in Fig. 10. The multipliers are marked as MUT with their corresponding twiddle-factor ROMs right beside, and the PEs for processing elements are labelled as Ux. Considering circuit overheads in SRAM, all delay lines longer than 64 are implemented by SRAM, while shorter ones are realised by registers. A brief summary of the chip is given in Table 4. There was an error made in some of the ROM values but, discounting that error, the rest of the chip can operate as designed. The FFT processor can operate up to $17.8 \mathrm{MHz}$ and dissipates $176 \mathrm{~mW}$ at $2.3 \mathrm{~V}$ supply voltage and it can operate up to $45 \mathrm{MHz}$ at $3.3 \mathrm{~V}$ supply voltage when it consumes $640 \mathrm{~mW}$.

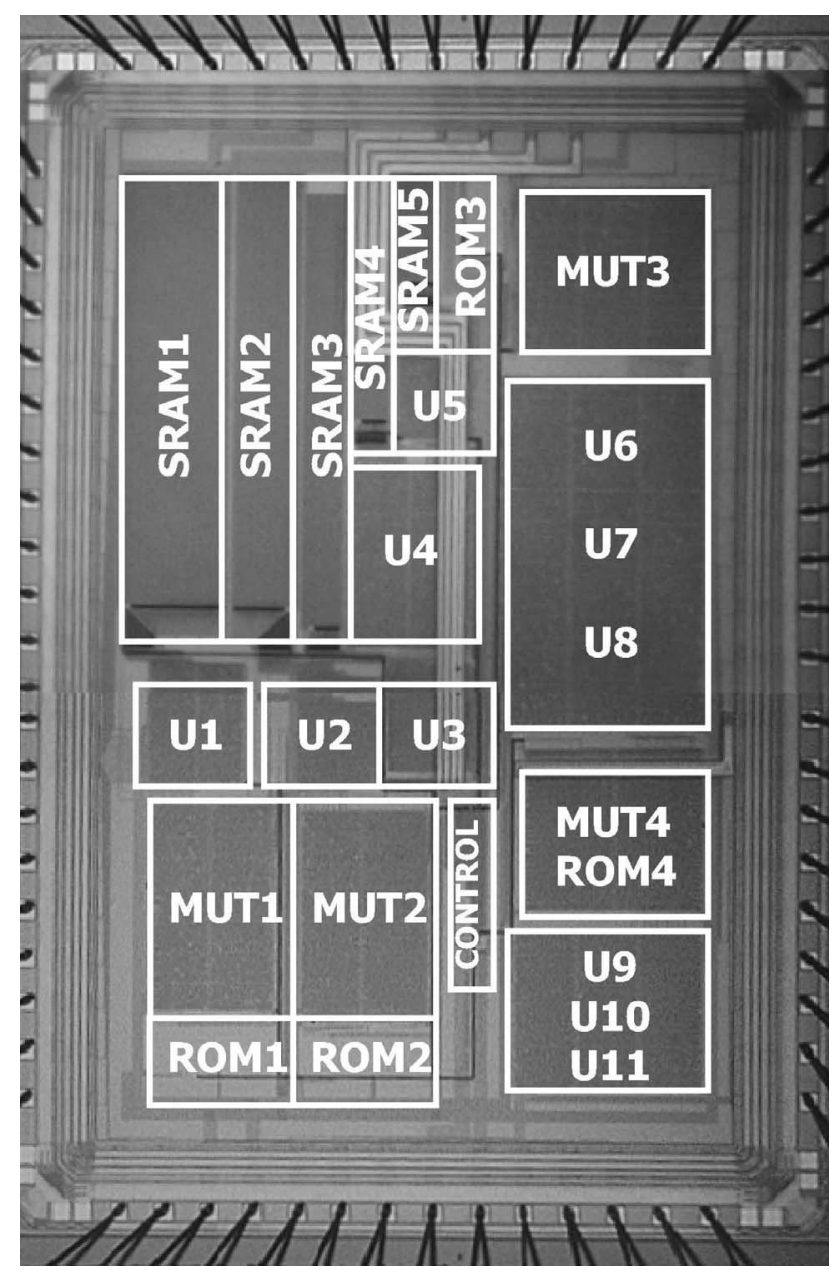

Fig. 10 Die photograph of proposed FFT processor
Comparisons of the proposed chip with several FFT processors [9, 17, 20, 21], including FFT size, algorithm, process, supply voltage, power consumption, clock rate, execution time and area, are listed in Table 5. Because these FFT processors are fabricated in different CMOS technologies and the FFT sizes are also different, it is not easy to make a fair comparison. We adopted three indices to make comparisons and adjusted the numbers by estimation assuming all processors perform a 1024-point FFT.

We use the normalised area, a metric in [21], and it is given by

$$
\text { Normalised area }=\frac{\text { Area of } 1024-\text { point FFT }}{(\text { Technology } / 0.35 \mu \mathrm{m})^{2}}
$$

to evaluate the cost of silicon. Next, we compare energy efficiency by FFT/Energy [21], which is given by

$$
\begin{aligned}
& \text { FFT } / \text { Energy }= \\
& \frac{\text { Technology }}{\text { Power of } 1024-\text { point FFT } \times \text { Execution Time } \times 10^{-6}}
\end{aligned}
$$

Another metric considering both energy efficiency and speed performance is the energy-time product, and it is given by

$$
\text { Energy } \times \text { Time }=\frac{\text { Execution Time }}{\text { FFT } / \text { Energy }}
$$

We can see from the Table that the proposed chip has the smallest normalised area and the smallest energy-time product. Although the FFT processor in [21] has the best energy efficiency when operating at $1.1 \mathrm{~V}$, its slow execution speed at that low voltage prevents it from highspeed applications in Table 1.

\section{Table 4: Chip summary}

\begin{tabular}{ll}
\hline Process & TSMC 0.35 1P4M \\
Area & $3.9 \mathrm{~mm} \times 5.5 \mathrm{~mm}$ \\
Transistor count & 598078 \\
Maximum frequency & $45 \mathrm{MHz}$ at $3.3 \mathrm{~V}$ \\
Power consumption (at highest & $640 \mathrm{~mW}$ (at $45 \mathrm{MHz}, 3.3 \mathrm{~V}$ ) \\
$\quad$ speed) & \\
Power consumption (at lowest & $176 \mathrm{~mW}$ (at $17.8 \mathrm{MHz}, 2.3 \mathrm{~V})$ \\
$\quad$ voltage) & \\
Package & $68 \mathrm{PGA}$
\end{tabular}


Table 5: Performance comparison of several FFT processors

\begin{tabular}{|c|c|c|c|c|c|c|c|c|}
\hline & Jia [9] & Bidet [20] & Baas [ & & & Li [17] & \multicolumn{2}{|c|}{ Proposed chip } \\
\hline FFT size $(N)$ & 8192 & 8192 & & \multicolumn{2}{|l|}{1024} & 1024 & \multicolumn{2}{|c|}{2048} \\
\hline Word length & 12 & 12 & \multicolumn{3}{|c|}{20} & N.A. & \multicolumn{2}{|c|}{12} \\
\hline Algorithm & $\operatorname{radix}-2 / 4 / 8$ & radix- 4 & \multicolumn{3}{|c|}{ radix-2 } & radix -4 & \multicolumn{2}{|c|}{$\operatorname{radix}-2 / 4 / 8$} \\
\hline Process, $\mu \mathrm{m}$ & 0.6 & 0.5 & \multicolumn{3}{|c|}{0.6} & 0.35 & \multicolumn{2}{|c|}{0.35} \\
\hline Voltage, V & 3.3 & 3.3 & 3.3 & 2.5 & 1.1 & 1.5 & 3.3 & 2.3 \\
\hline Power, mW & 650 & 300 & 845 & 363 & 9.5 & 200 & 640 & 176 \\
\hline 1024-pt power, mW & 435 & 300 & 845 & 363 & 9.5 & 200 & 480 & 132 \\
\hline Clock rate, $\mathrm{MHz}$ & 20 & 20 & 173 & 128 & 16 & 25 & 45.45 & 17.86 \\
\hline 1024-pt. exec. time, $\mu \mathrm{s}$ & 51 & 51 & 30 & 41 & 330 & 40 & 22.5 & 57 \\
\hline Area, $\mathrm{mm}^{2}$ & 107 & 100 & \multicolumn{3}{|c|}{36} & 10.54 & \multicolumn{2}{|c|}{13.05} \\
\hline Normalised area & 24.39 & 32.83 & & 12.25 & & 10.54 & \multicolumn{2}{|c|}{10.44} \\
\hline FFT/energy & 26.88 & 32.68 & 23.67 & 40.31 & 191.39 & 43.75 & 32.41 & 46.52 \\
\hline Energy $\times$ time & 1.91 & 1.56 & 1.27 & 1.02 & 1.72 & 0.91 & 0.69 & 1.23 \\
\hline
\end{tabular}

\section{Conclusion}

In this paper, we have reported the design of an FFT processor chip that is suitable for OFDM communication systems, such as DAB, DVB-T, ADSL and VDSL, for performing complex FFTs of lengths 512/1024/2048. The proposed variable-length FFT processor not only achieves efficient hardware utilisation but also low power consumption. The single-path delay feedback FFT architecture requires fewer delay elements and the radix-2/4/8 FFT algorithm replaces some complex multipliers with shiftand-add operations. In addition, some other circuit techniques have been applied for saving complexity as well as power consumption. The chip was implemented using a $0.35 \mu \mathrm{m}$ CMOS process. The measured results show that the chip can operate up to $45 \mathrm{MHz}$ under a $3.3-\mathrm{V}$ supply voltage and it consumes $640 \mathrm{~mW}$. When the supply voltage is scaled down to $2.3 \mathrm{~V}$, this processor consumes only $176 \mathrm{~mW}$ when it runs at $17.8 \mathrm{MHz}$.

\section{Acknowledgments}

The authors wish to thank the anonymous reviewers for their comments, which have that greatly improved this paper. Chip fabrication through the National Chip Implementation Center in Taiwan is also greatly appreciated. This work was supported in part by the National Science Council, Taiwan, ROC, under grant nos. NSC92-2220-E-002-002 and NSC92-2219-E-002-009 and by the MOE Program for Promoting Academic Excellence of the Universities under grant no. 89E-FA06-2-4-7.

\section{References}

1 Bingham, J.A.C.: 'Multicarrier modulation for data transmission: an idea whose time has come', IEEE Commun. Mag., 1990, 28, (7), pp. $5-14$

2 Cimini, L.J.: 'Analysis and simulation of a digital mobile channel using orthogonal frequency division multiplexing', IEEE Trans. Commun., $1985,33,(7)$, pp. $665-675$
3 ETSI EN 300401 (v1.3.2): 'Radio broadcasting systems; digital audio broadcasting (DAB) to mobile, portable and fixed receivers', Sep. 2000

4 ETSI EN 300744 (v1.2.1): 'Digital video broadcasting (DVB); framing structure, channel coding and modulation for digital terrestrial television', Jul. 1999

5 T1E1.4/98-007R4: 'Standards project for interfaces relating to carrier to customer connection of asymmetrical digital subscriber line (ADSL) equipment', Jun. 1998

6 ETSI TS 101 270-2 (V1.1.1): 'Transmission and multiplexing (TM); access transmission systems on metallic access cables; very high speed digital subscriber line (VDSL); Part 2: Transceiver specification', Feb. 2001

7 Rabiner, L.R., and Gold, B.: 'Theory and application of digital signal processing' (Prentice-Hall, Inc., NJ, 1975)

8 Groginsky, H.L., and Works, G.A.: 'A pipeline fast Fourier transform', IEEE Trans. Comput., 1970, 19, (11), pp. 1015-1019

9 Jia, L., Gao, Y., Isoaho, J., and Tenhunen, H.: 'A new VLSI-oriented FFT algorithm and implementation'. Proc. IEEE ASIC Conf., 1998 pp. $337-341$

$10 \mathrm{He}, \mathrm{S}$., and Torkelson, M.: 'Designing pipeline FFT processor for OFDM (de)modulation'. Proc. IEEE URSI Int. Symp. Signals, Systems and Electronics, 1998, pp. 257-262

11 Hui, C.C.W., Ding, T.J., and McCanny, J.V.: 'A 64-point Fourier transform chip for video motion compensation using phase correlation', IEEE J. Solid-State Circuits, 1996, 31, pp. 1751-1761

12 Chang, Y.-N., and Parhi, K.K.: 'An efficient pipelined FFT architecture', IEEE Trans. Circuits Syst. II, Analog Digit. Signal Process, 2003, 50, (6), pp. $322-325$

$13 \mathrm{Hu}$, Y.H.: 'CORDIC based VLSI architecture for digital signal processing', IEEE Signal Process. Mag., 1992, (4), pp. 16-35

14 Sarmiento, R., Tobajas, F., de Armas, V., Esper-Chain, R., Lopez, J.F., Montiel-Nelson, J.A., and Nunez, A.: 'A CORDIC processor for FFT computation and its implementation using gallium arsenide technology', IEEE Trans. VLSI Syst., 1998, 6, (1), pp. 18-30

15 Wenzler, A., and Luder, E.: 'New structures for complex multipliers and their noise analysis'. Proc. IEEE Int. Symp. on Circuits and Systems, May 1995, Vol. 2, pp. 1432-1435

$16 \mathrm{Hu}$, Y.H.: 'The quantization effects of the CORDIC algorithm', IEEE Trans. Signal Process., 1992, 40, (4), pp. 834-844

$17 \mathrm{Li}, \mathrm{W}$, and Wanhammer, L.' 'A pipeline FFT processor', Proc. Workshop Signal Processing Systems Design and Implementation, 1999, pp. 654-662

18 Wang, J.-S., Tseng, W., and Li, H.-Y.: 'Low-power embedded SRAM with the current-mode write technique', IEEE J. Solid-State Circuits, 2000, 35, (1), pp. 119-124

19 Tan, L.K., and Samueli, H.: 'A $200 \mathrm{MHz}$ quadrature digital synthesizer/mixer in $0.8 \mu \mathrm{m}$ CMOS', IEEE J. Solid-State Circuits, 1995, 30, (3), pp. 193-200

20 Bidet, E., Castelain, D., Joanblanq, C., and Senn, P.: 'A fast single-chip implementation of 8192 complex point FFT', IEEE J. Solid-State Circuits, 1995, 30, (3), pp. 300-305

21 Baas, B.M.: 'A low-power, high-performance, 1024-point FFT processor', IEEE J. Solid-State Circuits, 1999, 34, (3), pp. 380-387 